\title{
Evaluation of glioma-associated oncogene 1 expression and its correlation with the expression of sonic hedgehog, E-cadherin and S100a4 in human hepatocellular carcinoma
}

\author{
XIN ZHENG, YINGMIN YAO, QIURAN XU, KANGSHENG TU and QINGGUANG LIU \\ Department of Hepatobiliary Surgery, The First Affiliated Hospital of the \\ Medical College of Xi'an Jiaotong University, Xi'an 710061, P.R. China
}

Received July 19, 2010; Accepted September 24, 2010

DOI: $10.3892 / \mathrm{mmr} .2010 .375$

\begin{abstract}
The aim of the present study was to analyze the mRNA and protein expression of glioma-associated oncogene 1 (Gli1) in hepatocellular carcinoma (HCC) and its correlation with sonic hedgehog (Shh), one of the ligands of hedgehog $(\mathrm{Hh})$ signaling, and two epithelial-mesenchymal transition (EMT) markers, E-cadherin and S100a4. We also investigated the potential crosstalk between $\mathrm{Hh}$ signaling and EMT in HCC. Paired HCC and normal tumor-adjacent tissues were freshly collected from 30 primary HCC patients. Glil expression at both the mRNA and protein level was examined by reverse transcription-polymerase chain reaction and immunohistochemistry. The protein expression of Shh, E-cadherin and S100a4 was evaluated by immunohistochemistry to identify correlations with Gli1. The mRNA and protein expression of Gli1 was significantly up-regulated in the HCC tumor tissues compared to the normal tumor-adjacent tissues $(\mathrm{P}<0.01$, respectively). Gli1 protein expression in $\mathrm{HCC}$ was closely correlated with liver cirrhosis $(\mathrm{r}=0.460, \mathrm{P}=0.011<0.05)$, intrahepatic metastases $(\mathrm{r}=0.399, \mathrm{P}=0.029<0.05)$, portal vein invasion $(\mathrm{r}=0.367, \mathrm{P}=0.046<0.05)$, high Edmonson-Steiner classification $(\mathrm{r}=0.391, \mathrm{P}=0.032<0.05)$ and advanced TNM stage $(\mathrm{r}=0.416, \mathrm{P}=0.022<0.05)$. In HCC tissues, Gli1 protein expression was positively correlated with Shh $(\mathrm{r}=0.584$, $\mathrm{P}=0.001<0.05)$ and S100a4 $(\mathrm{r}=0.49, \mathrm{P}=0.006<0.05)$, and negatively correlated with E-cadherin $(\mathrm{r}=-0.439, \mathrm{P}=0.015<0.05)$. Glil was found to be up-regulated in HCC tissues and closely correlated with clinicopathological characteristics, indicating the enhanced metastatic potential of HCC. Furthermore, the increased expression of Gli1 in HCC cells may be attributed to Shh produced aberrantly by the cells themselves. Finally, Gli1
\end{abstract}

Correspondence to: Dr Qingguang Liu, Department of Hepatobiliary Surgery, the First Affiliated Hospital of Medical College of Xi'an Jiaotong University, Xi'an 710061, P.R. China

E-mail: raymondzhengxin@foxmail.com

Key words: hepatocellular carcinoma, glioma-associated oncogene 1, sonic hedgehog, S100a4 may play an important role in HCC invasion and metastasis by inducing EMT.

\section{Introduction}

Hepatocellular carcinoma (HCC) is the fifth most common cancer and the third leading cause of cancer-related death worldwide (1). Despite recent advances in the diagnosis and treatment of HCC, its mortality rate remains high, since HCC is associated with a high potential for vascular invasion and metastasis. Even after potentially curative hepatic resection, intrahepatic and extrahepatic metastases occur in more than $50 \%$ of patients and, among these, intrahepatic metastases appear more frequently (2). The diagnosis and prevention of $\mathrm{HCC}$ metastases or recurrence as soon as possible is therefore critical for HCC therapy.

Metastasis is a complex process, and various factors are involved at each step (3). HCC metastasis is related to the ability of tumor cells to invade the capsule and portal vein. Epithelial-mesenchymal transition (EMT) is a key event in the cancer metastasis process, whereby epithelial cells (or cancer cells) switch from an apical-basolateral, polarized epithelial phenotype to a spindle-shaped, fibroblast-like mesenchymal phenotype (4). Owing to the dramatic changes, cancer cells undergoing EMT easily dissociate from epithelial tissue (or primary malignancy) and migrate to other organs. The key feature in the initiation and execution of EMT is the downregulation of epithelial markers such as E-cadherin (5), and the up-regulation of mesenchymal markers such as S100a4 (6). Studies have confirmed that EMT frequently occurs in HCC and is involved in tumorigenesis and metastasis (7-9). Nevertheless, the mechanism underlying the initiation of EMT in $\mathrm{HCC}$ remains obscure.

The hedgehog (Hh) signaling pathway is critical during embryonic development and is involved in the patterning of the gastrointestinal tract, including the liver (10). However, this pathway did not appear to retain function in adult liver tissue due to the fact that mature hepatocytes lack $\mathrm{Hh}$ pathway activity (11). Recently, research on Hh signaling and $\mathrm{HCC}$ revealed that sonic hedgehog (Shh), a ligand of the $\mathrm{Hh}$ signaling pathway, is expressed aberrantly in HCC, meaning that Hh signaling may play a crucial role in the pathologic 
progression of HCC. Notably, evidence indicates crosstalk between $\mathrm{Hh}$ signaling and EMT in gastric cancer, and $\mathrm{Hh}$ signaling regulates EMT during human biliary fibrosis; in addition, Shh is the pivotal ligand or inducer during both mechanisms. Glioma-associated oncogene 1 (Gli1) is the most important Hh signaling downstream transcription factor and regulates the transcription of numerous target genes, including the EMT regulators SIP1, ZEB2 and ZFHX1B (12). In light of the above findings, we hypothesized that there may be aberrant activation of Shh/Glil signaling regulating the process of EMT in HCC. To investigate this hypothesis, we initially detected the expression and clinical significance of Gli1 in 30 HCC patients by reverse transcription-polymerase chain reaction (RT-PCR) and immunohistochemistry, and evaluated its correlation with the expression of Shh, E-cadherin and S100a4 at the protein level.

\section{Materials and methods}

HCC tissue and matched normal HCC-adjacent tissue specimens. A total of 30 patients with HCC were enrolled in the study between January 2009 and October 2009, including 16 men and 14 women (mean age 52 years, range 35-71) who had not received pre-operative chemotherapy or embolization. After routine X-ray, abdominal ultrasonography and computed tomography, all patients underwent liver resection, including curative resection for early $\mathrm{HCC}$ and palliative resection for advanced HCC. Tumor tissue and matched normal tumoradjacent tissue specimens $(>2 \mathrm{~cm}$ distance to the resection margin) were collected and immediately stored in liquid nitrogen for RT-PCR and paraformaldehyde for immunohistochemistry, respectively. Clinical data were obtained from the medical records. The histopathologic Edmonson classification, clinical tumor-node-metastasis (TNM) grading, maximum tumor diameter and the normal tumor-adjacent tissues were all confirmed by an experienced pathologist who was blinded to the clinical information.

Written informed consent was obtained from all patients. The Xi'an Jiaotong University Ethics Committee approved all protocols according to the Helsinki Declaration of 1975.

RT-PCR. RT-PCR was performed on frozen specimens stored in liquid nitrogen. The RNAfast 200 purification (Shanghai Fastagen Biotech Ltd. Co., China), PrimeScript RT reagent (Takara Bio Inc., Japan) and 2X Taq MasterMix (Beijing CoWin Biotech Ltd. Co., China) kits were used. Gli1 primers for RT-PCR, designed by Beacon Designer software (Premier Biosoft International, Palo Alto, CA, USA), were: 5'-TCATGTCCGGCCCCCACAGT-3', 5'-GTCCAGGCTGGC ATCCGACA-3'; the PCR product was $146 \mathrm{bp}$. GAPDH, a housekeeping gene, served as an internal control to ensure that an equal amount of mRNA was analyzed from each sample. The upstream primer sequence for GAPDH was 5'-GAAGGTGAAGGTCGGAGT-3' and the downstream sequence was 5'-GAAGATGGTGATGGGATTTC-3', which were expected to produce a 258-bp PCR product.

The total RNA of the samples was extracted in accordance with the manufacturer's instructions included in the RNAfast 200 purification kit. The optical density at A260/280 nm of RNA was between 1.7 and 1.9. The integrity of the RNA was confirmed by the presence of intact $18 \mathrm{~S}$ and $28 \mathrm{~S}$ bands on $1 \%$ agarose gel. Then, total RNA was reverse-transcribed at $37^{\circ} \mathrm{C}$ for $15 \mathrm{~min}$, and cDNA was incubated at $85^{\circ} \mathrm{C}$ for $5 \mathrm{sec}$ to inactivate the reverse transcriptase. For PCR amplification, cDNA was used as the template to amplify specific PCR products of the Gli1 and GAPDH genes. The PCR reaction was performed in a $25-\mu 1$ system, starting with denaturation at $94^{\circ} \mathrm{C}$ for $2 \mathrm{~min}$; then 35 cycles of denaturation at $94^{\circ} \mathrm{C}$ for $30 \mathrm{sec}$; annealing at $60^{\circ} \mathrm{C}$ and $55^{\circ} \mathrm{C}$ for Gli1 and GAPDH, respectively, for $30 \mathrm{sec}$; and extension at $72^{\circ} \mathrm{C}$ for $30 \mathrm{sec}$, followed by an additional extension at $72^{\circ} \mathrm{C}$ for $5 \mathrm{~min}$. The PCR products were separated by $1 \%$ agarose gel electrophoresis and stained with ethidium bromide. The electrophoresis bands were analyzed with the Gene Genius Gel Imaging System. Gene expression intensity (relative coefficient) was quantitatively analyzed by the ratio of the expression intensity of the electrophoresis bands to GAPDH

Immunohistochemical staining. Primary goat anti-Glil and primary goat anti-Shh antibodies were obtained from Santa Cruz Biotechnology Inc. (USA). The primary mouse anti-Ecadherin antibody and primary rabbit anti-S100a4 were both obtained from Zhongshan Goldenbridge Biotechnology Ltd. Co. (China).

Immunohistochemistry was performed on paraformaldehyde-fixed paraffin sections. The sections were dewaxed and dehydrated. After rehydration, endogenous peroxidase activity was blocked for $30 \mathrm{~min}$ using a methanol solution containing $0.3 \%$ hydrogen peroxide. After antigen retrieval in citrate buffer, the sections were blocked overnight at $4^{\circ} \mathrm{C}$, then separately incubated with the primary antibodies directed against Gli1, Shh, E-cadherin and S100a4 at $4^{\circ} \mathrm{C}$ overnight. The primary antibody was detected using biotinylated secondary antibodies (Zhongshan Goldenbridge Biotechnology Ltd. Co.) according to the manufacturer's recommendations. The staining of the sections was performed using the avidinbiotin-peroxidase complex for Gli1 and Shh (SABC method) and HRP-streptavidin conjugates for E-cadherin and S100a4 (SP method). The sections were visualized with diaminobenzidine and counterstained with hematoxylin, then dehydrated in alcohol and xylene and mounted onto glass slides.

All sections were assessed independently by two experienced pathologists. The staining results for the four proteins (Gli1, Shh, E-cadherin and S100a4) were semi-quantitatively expressed by an immunohistochemical score combined with the percentage of tumor cells showing specific immunoreactivity. Staining intensity was expressed as four grades: 0, none; 1, weak; 2, moderate; and 3, strong. The percentage of positive carcinoma cells was expressed as the following grades: $0,<5 \%$; $1,6-25 \% ; 2,26-50 \% ; 3,51-75 \%$; and $4,>75 \%$. The staining intensity and average percentage of positive tumor cells were assayed for 10 independent high magnification (x400) fields. The total score was calculated by multiplying the staining intensity and the percentage of positive tumor cells. Sections with a total score of $>1$ were defined as exhibiting positive staining for the above four proteins.

Statistical analysis. Data were expressed as the mean \pm SD. Differences in Gli1 mRNA and protein expression in the HCC tissues compared to the normal tumor-adjacent tissues 


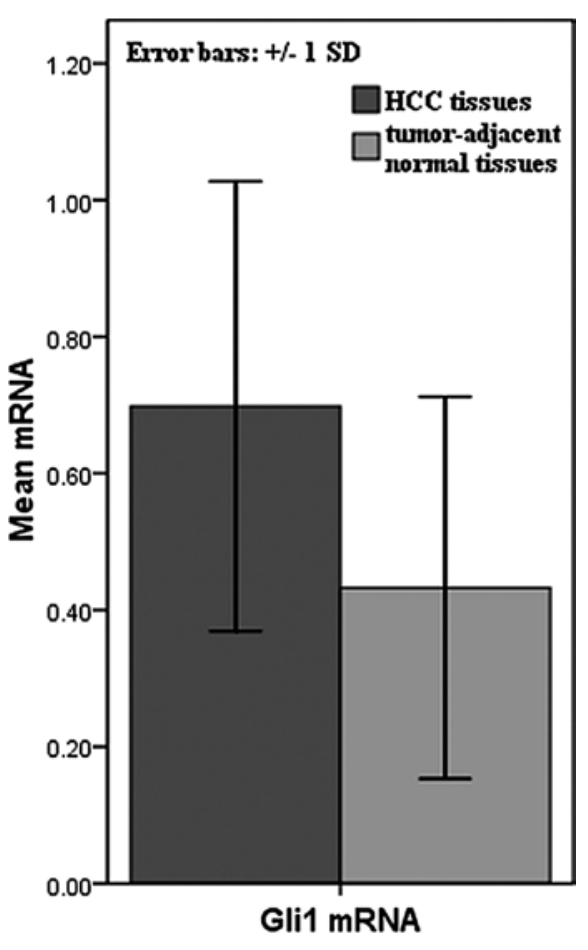

Figure 1. mRNA expression of Gli1 in the $30 \mathrm{HCC}$ patients.

were compared by the Mann-Whitney $U$ test. Since the immunopositivity scores of Gli1 protein were ranked data, the relationship between the clinical characteristics of the patients and Glil protein expression was analyzed using the Spearman rank test. Additionally, the Spearman rank test was also used to analyze the correlation between the Gli1 protein expression and Shh, E-cadherin and S100a4. Values of $\mathrm{P}<0.05$ were considered to be statistically significant. For all statistical analyses, the software package SPSS 13.0 (SPSS Inc., USA) was used.

\section{Results}

Expression of Glil in the $30 \mathrm{HCC}$ and matched normal tumoradjacent tissues. After analysis of the results of RT-PCR, the expression of Gli1 mRNA in the HCC tissues was found to be significantly higher than that in the matched normal tumoradjacent tissues $(0.70 \pm 0.06$ vs. $0.43 \pm 0.28 ; \quad \mathrm{P}=0.002<0.05)$ (Fig. 1). Most of the positive HCC cells showed diffuse cytoplasmic staining of Gli1, while nuclear staining was found in a few HCC cells. Additionally, corroborating the results at the mRNA level, immunohistochemistry revealed that the expression of Gli1 protein was significantly higher in the HCC tissues than in the matched normal tumor-adjacent tissues (5.27 \pm 2.94 vs. $2.40 \pm 2.00 ; \mathrm{P}<0.001)$ (Figs. 2 and 3).

Correlation between Glil protein expression in the 30 HCC tissues and clinicopathological characteristics. The correlation between Glil protein expression and the clinicopathological characteristics of the $30 \mathrm{HCC}$ patients, was statistically analyzed, and the results are listed in Table I. Gli1 protein expression was significantly correlated with liver cirrhosis $(r=0.460, P=0.011<0.05)$, intrahepatic metastases $(\mathrm{r}=0.399, \mathrm{P}=0.029<0.05)$, portal vein invasion $(\mathrm{r}=0.367$,

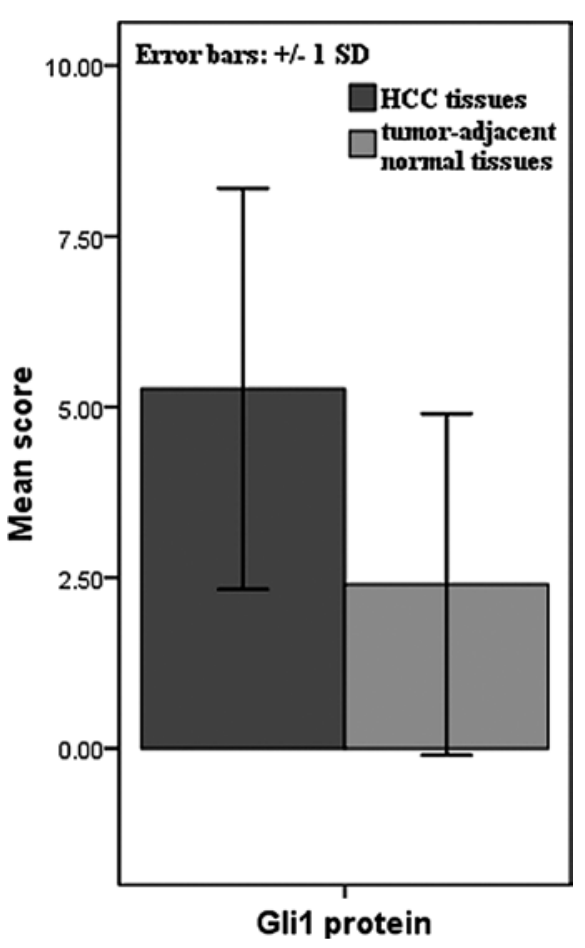

Figure 2. Protein expression of Gli1 in the $30 \mathrm{HCC}$ patients.

Table I. Correlation between the clinicopathological characteristics and protein expression of Gli1 in the $30 \mathrm{HCC}$ patients.

\begin{tabular}{lrc}
\hline Characteristics & r-value & P-value \\
\hline Gender & -0.040 & $0.835>0.05$ \\
Age & 0.115 & $0.544>0.05$ \\
HBV infection & 0.190 & $0.315>0.05$ \\
Liver cirrhosis & 0.460 & $0.011<0.05$ \\
High serum AFP level & 0.186 & $0.324>0.05$ \\
(>400 ng/ml) & & \\
Tumor size ( $>5$ cm) & -0.219 & $0.244>0.05$ \\
Intrahepatic metastases & 0.399 & $0.029<0.05$ \\
Portal vein invasion & 0.367 & $0.046<0.05$ \\
High Edmonson-Steiner & 0.391 & $0.032<0.05$ \\
classification (II and III) & & \\
Advanced TNM stage (III and IV) & 0.416 & $0.022<0.05$ \\
\hline
\end{tabular}

Table II. Protein expression of Shh, E-cadherin and S100a4 in the $30 \mathrm{HCC}$ patients (mean $\pm \mathrm{SD}$ ).

\begin{tabular}{lccc}
\hline & HCC tissues & $\begin{array}{c}\text { Normal tumor- } \\
\text { adjacent tissues }\end{array}$ & P-value \\
\hline Shh & $6.20 \pm 3.29$ & $2.73 \pm 2.75$ & $<0.001$ \\
E-cadherin & $1.03 \pm 1.81$ & $3.17 \pm 2.64$ & $0.001<0.05$ \\
S100a4 & $4.40 \pm 3.68$ & $0.4667 \pm 1.01$ & $<0.001$ \\
\hline
\end{tabular}

$\mathrm{P}=0.046<0.05)$, high Edmonson-Steiner classification $(\mathrm{r}=0.391, \mathrm{P}=0.032<0.05)$ and advanced TNM stage $(\mathrm{r}=0.416$, 

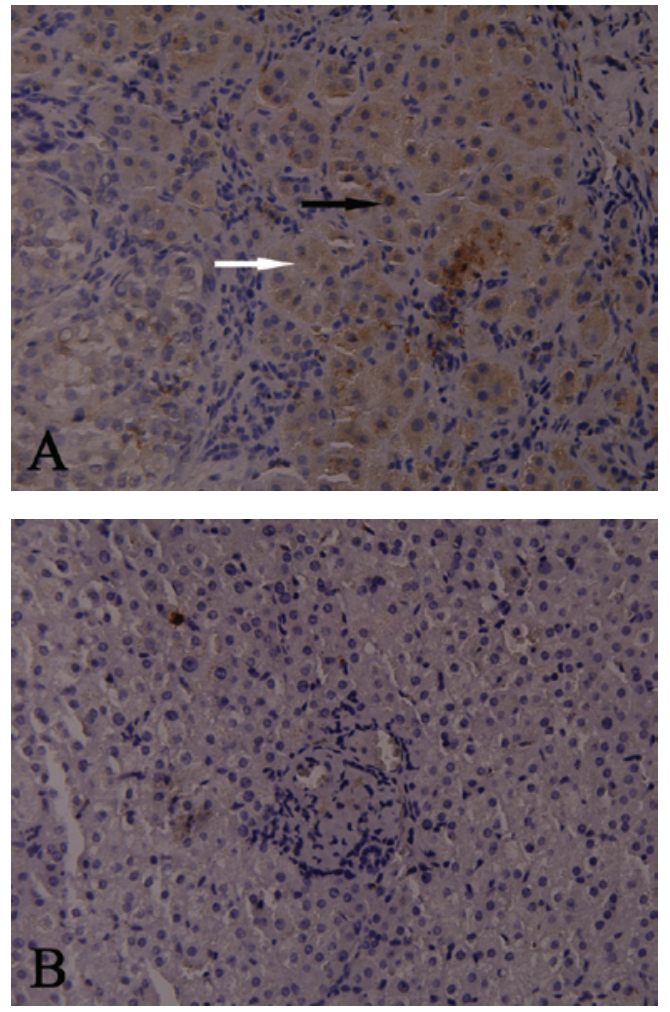

Figure 3. Immunohistochemical staining of Gli1 in (A) HCC tissue and (B) normal tumor-adjacent normal tissue. Gli1 protein expression in the HCC tissues was higher than that in the normal tumor-adjacent tissues. Most of the positive tumor cells showed cytoplasmic staining (white arrow), while nuclear staining was found in a few positive tumor cells (black arrow) Original magnification, $\mathrm{x} 400$.
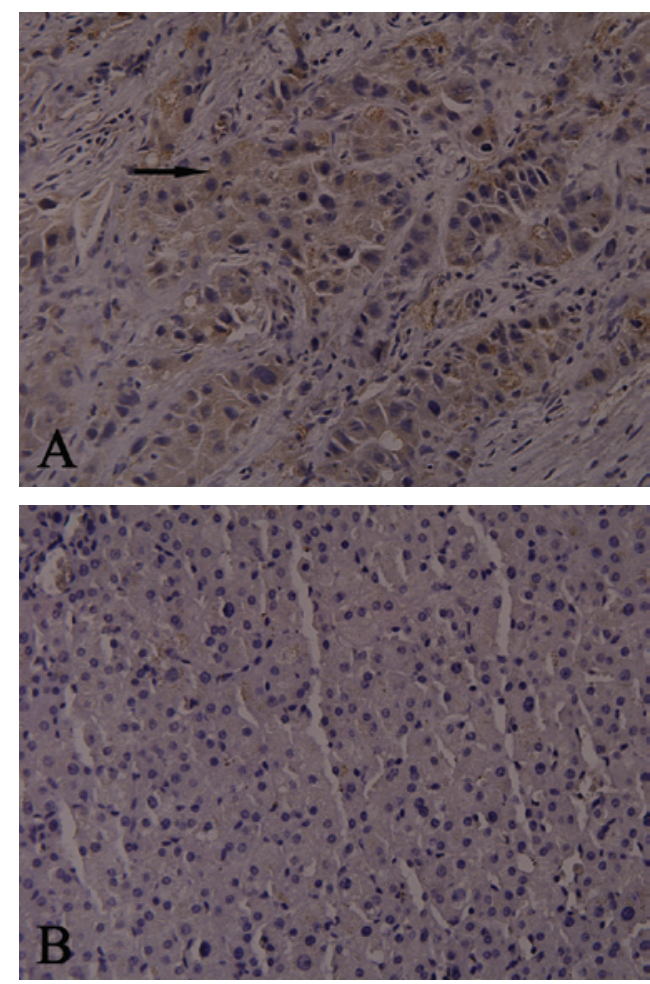

Figure 4. Immunohistochemical staining of Shh in (A) HCC tissue and (B) normal tumor-adjacent tissue. Shh protein expression in the HCC tissues was located mainly in the cytoplasm (black arrow) and was significantly higher than in the normal tumor-adjacent tissues. Original magnification, $\mathrm{x} 400$.
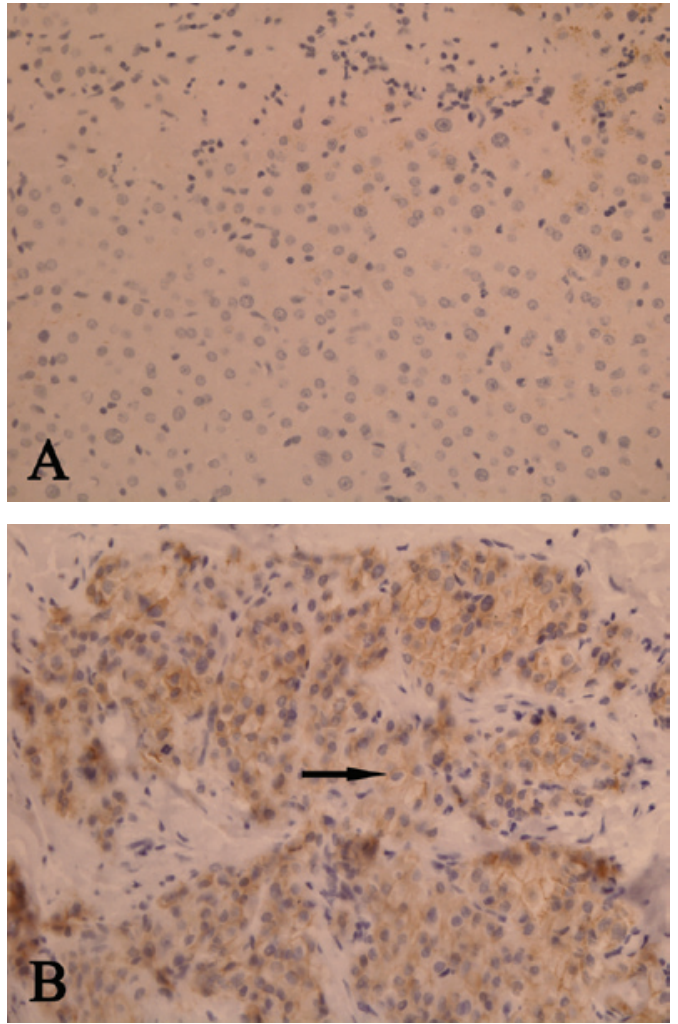

Figure 5. Immunohistochemical staining of E-cadherin in (A) HCC tissue and (B) normal tumor-adjacent tissue. E-cadherin protein expression in the normal tumor-adjacent tissues was significantly higher than that in the HCC tissues, where it was located mainly in the cytomembrane (black arrow). Original magnification, $\mathrm{x} 400$.
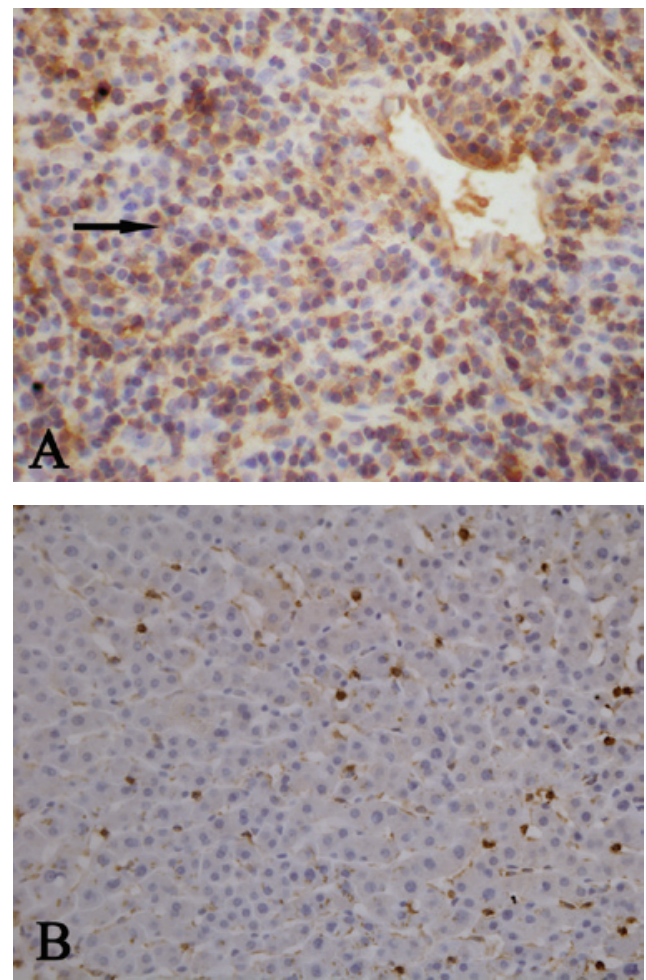

Figure 6. Immunohistochemical staining of S100a4 in (A) HCC tissue and (B) normal tumor-adjacent tissue. S100a4 protein expression in the HCC tissues was significantly higher than in the normal tumor-adjacent tissues, where it located mainly in the cytoplasm (black arrow). Original magnification, $\mathrm{x} 400$. 
$\mathrm{P}=0.022<0.05)$. However, no significant correlation was found between Gli1 protein expression and gender, age, HBV infection, serum $\alpha$-fetoprotein (AFP) level and tumor size.

Expression of Shh, E-cadherin and S100a4 in the $30 \mathrm{HCC}$ and matched normal tumor-adjacent tissues. The protein expression of Shh, E-cadherin and S100a4 was assessed semi-qualitatively by immunohistochemical analysis, and the results are listed in Table II. Both Shh and S100a4 proteins were localized mainly in the cytoplasm and were significantly elevated in the HCC tissues $(\mathrm{P}<0.001)$ (Figs. 4 and 5), while the E-cadherin protein was located in the cytomembrane and was significantly down-regulated in the HCC tissues $(\mathrm{P}=0.001<0.05)$ (Fig. 6).

Correlation between protein expression of Glil and Shh in the 30 HCC tissues. As mentioned above, both Glil and Shh protein expression were significantly higher in the HCC tissues than in the normal tumor-adjacent tissues. After analysis using the Spearman rank test, a significant positive correlation was noted between Gli1 and Shh at the protein level $(r=0.584$, $\mathrm{P}=0.001<0.05)$ in the HCC tissues.

Correlation between protein expression of Glil and E-cadherin in the $30 \mathrm{HCC}$ tissues. Glil protein expression was found to be significantly higher in the $30 \mathrm{HCC}$ tissues than that in the matched normal tumor-adjacent tissues, while the protein expression of E-cadherin was significantly downregulated in the $30 \mathrm{HCC}$ tissues. Addtionally, the level of Gli1 protein was negatively correlated with that of E-cadherin in the HCC tissues, as assessed by the Spearman rank test $(\mathrm{r}=-0.439, \mathrm{P}=0.015<0.05)$.

Correlation between protein expression of Glil and S100a4 in the $30 \mathrm{HCC}$ tissues. S100a4 protein was significantly increased in the $30 \mathrm{HCC}$ tissues, which was similar to the results of Gli1 protein expression. Moreover, a significant positive correlation was found between Gli1 and S100a4 protein expression $(r=0.49, \mathrm{P}=0.006<0.05)$.

Correlation between protein expression of E-cadherin and S100a4 in the 30 HCC tissues. E-cadherin was markedly down-regulated in the 30 HCC tissues, while S100a4 was up-regulated in the HCC tissues. In addition, a significant negative correlation between the protein expression of E-cadherin and S100a4 was confirmed in the HCC tissues $(\mathrm{r}=-0.767, \mathrm{P}<0.001)$ by the Spearman rank test.

\section{Discussion}

Hh signaling is well known to be essential for multiple aspects of embryonic development. Due to the fact that it is involved in organ patterning, cell differentiation and cell proliferation, Hh signaling has been considered to play a key role in the patterning of the neural tube, lung, skin, axial skeleton and gastrointestinal tract (13-16). However, in adults Hh signaling is mainly quiescent, and its inappropriate reactivation has been linked to diverse human disease entities, such as medulloblastoma, basal cell carcinoma, lung and gastric cancer (17). The transcription factor Gli1, a member of the Gli1 subfamily of zinc finger proteins, plays an important role in signal transduction within the Hh pathway. In previous studies, the aberrant activation of Glil was found to lead to the transcription of the Hh target genes involved in tumorigenesis and metastasis through Gli1 binding to the GACCACCCA motif, including Bcl2, D-type cyclins, VEGF and Snail. Additionally, Gli1, which is also one of the Hh target genes, amplifies the initial $\mathrm{Hh}$ signal at the transcriptional level; therefore, Gli1 is a reliable indicator of Hh signaling activity.

This study demonstrated that the mRNA and protein expression of Gli1 in HCC tissues was significantly higher than that in normal tumor-adjacent tissues, and the overexpression of Gli1 was significantly correlated with intrahepatic metastases, portal vein invasion, advanced TNM stage and other clinical characteristics. This finding implies that Glil may be involved in HCC metastasis. In previous studies, the $\mathrm{Hh}$ pathway was found to remain quiescent in adult liver tissue; however, in the present study, Glil was found to be activated not only in HCC tissues, but also in the normal tumor-adjacent tissues. Notably, the aberrant expression of Gli1 in the normal tumor-adjacent tissues was mainly observed in patients with liver cirrhosis, and the expression of Glil in HCC tissues was significantly correlated with liver cirrhosis. Thus, Gli1 may be involved in the pathogenesis of chronic liver injury, which induces liver cirrhosis, consistent with the results of Omenetti and Diehl (10).

The results regarding Glil expression in $\mathrm{HCC}$ patients revealed that Hh signaling is activated in HCC. Recent studies $(18,19)$ have concluded that there are three basic models proposed for $\mathrm{Hh}$ signaling activity in cancer: Type 1, Hh ligand-independent; Type 2, Hh ligand-dependent and autocine; and Type 3, Hh ligand-dependent and paracrine. In the present study, Shh expression was detected in the same HCC patients and Shh expression in HCC tissues was found to be significantly higher than that in the normal tumor-adjacent tissues, and Shh expression was correlated with Gli1 expression in the HCC tissues. This suggests that the activation of Hh signaling in $\mathrm{HCC}$ may be attributed to the fact that $\mathrm{HCC}$ cells respond to Shh, which is produced by the same tumor cells (Type 3). However, a previous study showed that hepatic stellate cells activated in liver cirrhosis also produced amounts of soluble Shh protein, which induced the activation of $\mathrm{Hh}$ signaling (20). Consequently, further research is required in order to elucidate the mechanism responsible for the activation of Hh signaling in HCC.

Potential relationship between Glil and EMT in HCC. The transition of epithelial cells to a mesenchymal phenotype (EMT) is of paramount importance for embryonic development and wound healing. Increasing evidence has shown that EMT mediates HCC progression, including local invasion, spreading through the circulation and metastasis (21). A hallmark of EMT is the loss of epithelial marker expression with aberrant expression of mesenchymal markers in cancer epithelial cells. In this study, we detected the expression of E-cadherin, a well-known epithelial marker, and S100a4, a mesenchymal marker, in $30 \mathrm{HCC}$ patients. We found that E-cadherin expression in the HCC tissues was down-regulated in comparison to that in the normal tumoradjacent tissues, while S100a4 was up-regulated in HCC 
tissues. Moreover, in the $30 \mathrm{HCC}$ patients enrolled in this study, a significant negative correlation was found between E-cadherin and S100a4. This implies that EMT may be involved in HCC progression. We next analyzed the correlation between E-cadherin, S100a4 and Gli1, respectively, using Spearman correlation analysis, and found a significant negative correlation between Gli1 and E-cadherin, and a significant positive correlation between Gli1 and S100a4. This demonstrates that Glil may be one of the inducers of EMT in HCC, and that there may be crosstalk between $\mathrm{Hh}$ signaling and EMT in HCC.

In conclusion, Gli1 was increased in HCC tissues and was correlated with clinicopathological characteristics related to invasion and metastasis. A negative correlation between E-cadherin and S100a4 was found. In addition, a significant negative correlation was noted between Glil and E-cadherin, while a significant positive correlation was found between Gli1 and S100a4. This suggests that Gli1 may regulate EMT in HCC. Shh was up-regulated in HCC tissues and was correlated with Gli1, suggesting that the activation of $\mathrm{Hh}$ signaling in $\mathrm{HCC}$ may be due to the fact that $\mathrm{HCC}$ cells respond to Shh, which was produced by the same tumor cells. However, due to the complex nature of the Hh pathway, more studies are required to elucidate the mechanism of activation of $\mathrm{Hh}$ signaling in HCC.

\section{Acknowledgements}

This study was supported by a grant from the National Natural Science Foundation of China (no. 81072052).

\section{References}

1. Parkin DM, Bray F, Ferlay J, et al: Global cancer statistics, 2002. CA Cancer J Clin 5: 74-108, 2005.

2. Poon RT, Fan ST and Wong J: Risk factors, prevention and management of postoperative recurrence after resection of hepatocellular carcinoma. Ann Surg 232: 10-24, 2000.

3. Eccles SA and Welch DR: Metastasis: recent discoveries and novel treatment strategies. Lancet 369: 1742-1757, 2007.

4. Liu Y and Dean DC: Tumor initiation via loss of cell contact inhibition versus Ras mutation: do all roads lead to EMT? Cell Cycle 9: 897-900, 2010.
5. Sanchez-Tillo E, Lazaro A, Torrent R, et al: ZEB1 represses E-cadherin and induces an EMT by recruiting the SWI/SNF chromatin-remodeling protein BRG1. Oncogene 29: 3490-3500, 2010.

6. Ghoul A, Serova M, Astorgues-Xerri L, et al: Epithelial-tomesenchymal transition and resistance to ingenol 3-angelate, a novel protein kinase $\mathrm{C}$ modulator, in colon cancer cells. Cancer Res 69: 4260-4269, 2009.

7. Van Zijl F, Zulehner G, Petz M, et al: Epithelial-mesenchymal transition in hepatocellular carcinoma. Future Oncol 5: 1169-1179, 2009.

8. Choi SS and Diehl AM: Epithelial-to-mesenchymal transitions in the liver. Hepatology 50: 2007-2013, 2009.

9. Jou J and Diehl AM: Epithelial-mesenchymal transitions and hepatocarcinogenesis. J Clin Invest 120: 1031-1034, 2010.

10. Omenetti A and Diehl AM: The adventures of sonic hedgehog in development and repair. II. Sonic hedgehog and liver development, inflammation, and cancer. Am J Physiol Gastrointest Liver Physiol 294: 595-598, 2008

11. Berman DM, Karhadkar SS, Maitra A, et al: Widespread requirement for Hedgehog ligand stimulation in growth of digestive tract tumours. Nature 425: 846-851, 2003.

12. Isohata N, Aoyagi K, Mabuchi T, et al: Hedgehog and epithelial-mesenchymal transition signaling in normal and malignant epithelial cells of the esophagus. Int J Cancer 125: 1212-1221, 2009.

13. Bian YH, Huang SH, Yang L, et al: Sonic hedgehog-Gli1 pathway in colorectal adenocarcinomas. World J Gastroenterol 13: 1659-1665, 2007.

14. Pons S and Marti E: Sonic hedgehog synergizes with the extracellular matrix protein vitronectin to induce spinal motor neuron differentiation. Development 127: 333-342, 2000.

15. Dessaud E, McMahon AP and Briscoe J: Pattern formation in the vertebrate neural tube: a sonic hedgehog morphogen-regulated transcriptional network. Development 135: 2489-2503, 2008.

16. Ramalho-Santos M, Melton DA and McMahon AP: Hedgehog signals regulate multiple aspects of gastrointestinal development. Development 127: 2763-2772, 2000.

17. Saqui-Salces $M$ and Merchant JL: Hedgehog signaling and gastrointestinal cancer. Biochim Biophys Acta 1803: 786-795, 2010.

18. Rubin LL and de Sauvage FJ: Targeting the Hedgehog pathway in cancer. Nat Rev Drug Discov 5: 1026-1033, 2006.

19. Scales SJ and de Sauvage FJ: Mechanisms of Hedgehog pathway activation in cancer and implications for therapy. Trends Pharmacol Sci 30: 303-312, 2009.

20. Lin N, Tang Z, Deng M, et al: Hedgehog-mediated paracrine interaction between hepatic stellate cells and marrow-derived mesenchymal stem cells. Biochem Biophys Res Commun 372: 260-265, 2008

21. Lee TK, Poon RT, Yuen AP, et al: Twist overexpression correlates with hepatocellular carcinoma metastasis through induction of epithelial-mesenchymal transition. Clin Cancer Res 12: 5369-5376, 2006. 